\title{
On the rotational alignment of graphene domains grown on $\mathrm{Ge}(110)$ and $\mathrm{Ge}(111)$
}

\author{
P.C. Rogge, Department of Materials Science and Engineering, University of California, Berkeley, Berkeley, CA 94720, USA; Materials Sciences Division, \\ Lawrence Berkeley National Laboratory, Berkeley, CA 94720, USA \\ M.E. Foster, Sandia National Laboratories, Livermore, CA 94550, USA \\ J.M. Wofford, Department of Materials Science and Engineering, University of California, Berkeley, Berkeley, CA 94720, USA; Materials Sciences Division, \\ Lawrence Berkeley National Laboratory, Berkeley, CA 94720, USA† \\ K.F. McCarty, and N.C. Bartelt, Sandia National Laboratories, Livermore, CA 94550, USA \\ O.D. Dubon, Department of Materials Science and Engineering, University of California, Berkeley, Berkeley, CA 94720, USA; Materials Sciences Division, \\ Lawrence Berkeley National Laboratory, Berkeley, CA 94720, USA
}

Address all correspondence to P. C. Rogge at progge@berkeley.edu

(Received 27 June 2015; accepted 31 August 2015)

\begin{abstract}
We have used low-energy electron diffraction and microscopy to compare the growth of graphene on hydrogen-free $\mathrm{Ge}(111)$ and $\mathrm{Ge}(110)$ from an atomic carbon flux. Growth on $\mathrm{Ge}(110)$ leads to significantly better rotational alignment of graphene domains with the substrate. To explain the poor rotational alignment on $\mathrm{Ge}(111)$, we have investigated experimentally and theoretically how the adatom reconstructions of Ge interact with graphene. We find that the ordering transition of the $\mathrm{Ge}(111)$ adatom reconstruction is not significantly perturbed by graphene. Density functional theory calculations show that graphene on reconstructed $\mathrm{Ge}(110)$ has large-amplitude corrugations, whereas it is remarkably flat on reconstructed $\mathrm{Ge}(111)$. We argue that the absence of corrugations prevents graphene islands from locking into a preferred orientation.
\end{abstract}

\section{Introduction}

Because of its intrinsic two-dimensional (2D) character, graphene interacts weakly with the substrates on which it is grown. Consequently, graphene domains tend to nucleate in random orientations, producing polycrystalline films. ${ }^{[1-5]}$ Much has been learned about the factors that control domain orientation, particularly on metal substrates. For example, it has been shown that a single, energetically preferred orientation exists on $\operatorname{Ir}(111)$, where the preferred graphene orientation is dictated by the amplitude of the moire corrugation of the graphene film induced by film-substrate interactions. ${ }^{[6]}$ Recently, germanium, a group IV semiconductor, has emerged as a viable substrate for graphene epitaxy. ${ }^{[7-9]}$ High quality epitaxial growth on semiconductor wafers is very attractive because it presents a path toward production by existing very large scale integration processes. Graphene growth on $\mathrm{Ge}(110)$ by chemical vapor deposition (CVD) has been shown to exhibit considerably improved rotational alignment compared with growth on $\mathrm{Ge}(111) \cdot{ }^{[7]}$ Here, we clarify the reasons for this improvement.

Due to the directional nature of covalent bonds, semiconductor surfaces undergo distinctive reconstructions to minimize

†Current Address: Paul-Drude-Institut für Festkörperelektronik, Berlin 10117, Germany. dangling bonds, which presents a heterogeneous bonding environment for graphene in contrast to closed-packed metal surfaces. In the case of germanium, the surface reconstructions consist of ordered adatom phases that exhibit first-order disordering phase transitions with temperature. ${ }^{[10,11]}$ The high-temperature phases are believed to be disordered versions of the low-temperature phases such that both contain similar densities of adatoms. ${ }^{[10]}$ It is critical to understand how graphene films interact with this complex growth substrate in order to optimize growth on germanium and other covalently bonded materials.

One way to probe the interaction between Ge and graphene, and thus to understand what dictates the degree of alignment, is to determine how the presence of graphene affects the Ge surface reconstruction - that is, how graphene affects the nature of the ordered or disordered adatom phases and the transition between them. This effect is a measure of how strongly the graphene is coupled to the substrate. To probe this effect, we have used low-energy electron microscopy (LEEM) and lowenergy electron diffraction (LEED) to study graphene growth on $\mathrm{Ge}(111)$ and $\mathrm{Ge}(110)$ while monitoring the nature of the surface reconstruction. We find that the clean $\mathrm{Ge}(111)$ surface reconstruction remains even when supporting a graphene film, whereas the $\mathrm{Ge}(110)$ surface reconstruction is more strongly affected. Further, we used density functional theory (DFT) to investigate the influence of the Ge surface reconstruction on the 
structure of the graphene film. We find that graphene on the adatom-reconstructed $\mathrm{Ge}(111)$ surface is remarkably flat compared to graphene on $\mathrm{Ge}(110)$, which we argue (following Ref. 6) explains the improved rotational order on $\mathrm{Ge}(110)$.

\section{Methods}

Germanium substrates were cut from single crystal wafers and prepared in the low-energy electron microscope by cycles of Ar-ion sputtering $\left(1.5 \mathrm{keV}, 5 \times 10^{-6}\right.$ torr $\mathrm{Ar}, 5 \mathrm{~min}$, base pressure $1 \times 10^{-10}$ torr) and annealing at $\sim 800^{\circ} \mathrm{C}$. Temperature was measured by a W-Rh thermocouple in contact with a tantalum disc supporting the Ge sample. Temperature readings were calibrated using the well-known transition temperatures of the surface reconstructions for $\mathrm{Ge}(111)^{[10]}$ and $\mathrm{Ge}(110) .^{[11]}$ In general, the thermocouple reading was $\sim 50 \mathrm{~K}$ less than the estimated actual sample temperature. Heating a sample until melting further confirmed this estimate. The temperatures reported here are adjusted and are estimated to be accurate to $\pm 20 \mathrm{~K}$. Carbon was deposited by physical vapor deposition (PVD), where an elemental carbon vapor flux was produced by heating a graphite rod by bombardment with $3 \mathrm{keV}$ electrons. After growth and in-situ characterization, samples were removed from the microscope and exposed to the ambient atmosphere for at least $24 \mathrm{~h}$. Samples were then placed in roomtemperature de-ionized water for $24 \mathrm{~h}$. A silicon wafer with a thick oxide layer was used to collect graphene floating on the water surface. The graphene was then dried by heating the wafer on a hot plate at $150{ }^{\circ} \mathrm{C}$ for $10 \mathrm{~min}$. Raman spectroscopy was used to evaluate the transferred graphene.

For all DFT calculations, the non-local optB86b-vdW (van der Waals) exchange-correlation functional ${ }^{[12]}$ was used within the unrestricted Kohn-Sham formalism. The projectoraugmented-wave method, as implemented in VASP (Vienna $\mathrm{Ab}$ initio simulation package), ${ }^{[13-16]}$ was utilized to model the core electrons, and the wave functions were expanded in a plane-wave basis with an energy cutoff of $400 \mathrm{eV}$. A gammapoint sampling of the Brillouin zone was used for all calculations except bulk Ge where a $10 \times 10 \times 10 \mathrm{k}$-point grid was used. The optB86b-vdW exchange-correlation functional was used for its ability to approximately account for dispersion interactions (van der Waals forces), which is imperative to qualitatively model graphene-substrate interactions. Moreover, it has been previously demonstrated that the optB86b-vdW functional is currently among the most accurate vdW functionals. ${ }^{[12,17,18]}$

\section{Results}

LEEM images were acquired in mirror-mode, where the incident electron energy ( $\sim 1 \mathrm{eV}$ used here) is less than the surface work function. In this mode, image contrast is sensitive to surface defects and changes in work function. ${ }^{[19,20]}$ After Ar-ion sputtering, the surface is featureless except for a low density of defects as seen in Fig. 1(a). Observable defects with diameter $\sim 700 \mathrm{~nm}$ were present in low density $\left(\sim 2 \times 10^{4} / \mathrm{cm}^{2}\right)$ as well as smaller defects, $\sim 200 \mathrm{~nm}$ in diameter, with higher density
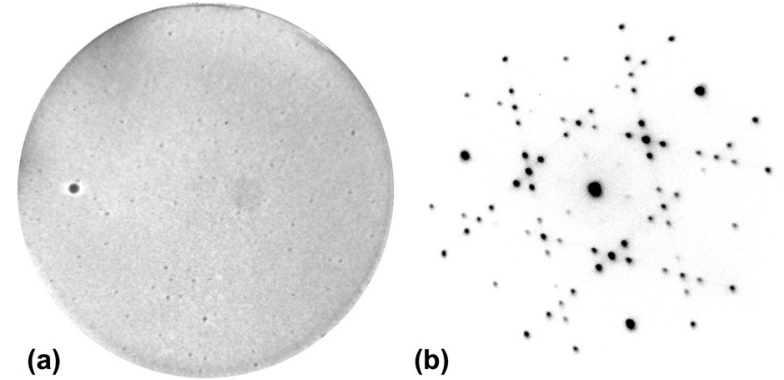

(a)

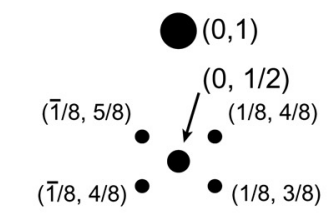

(b)
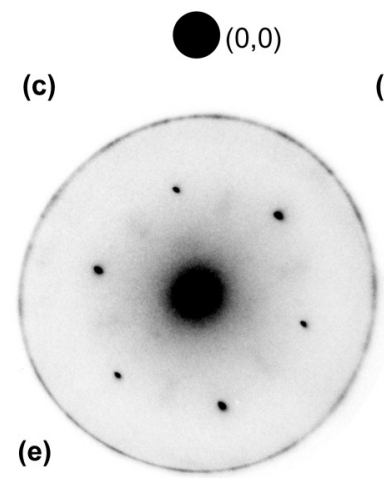

(d)
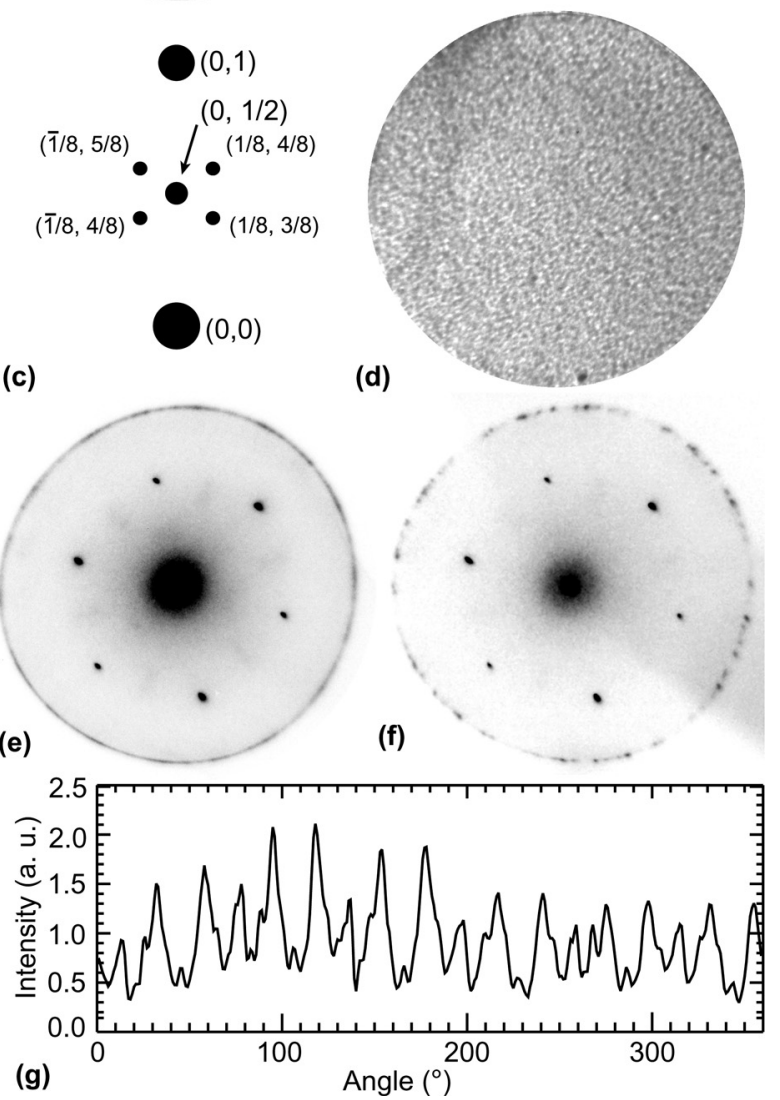

Figure 1. (a) Initial $\mathrm{Ge}(111)$ surface after sputtering, field-of-view $(\mathrm{FOV})=25 \mu \mathrm{m}$, $T=25^{\circ} \mathrm{C}$. (b) LEED pattern of initial surface showing $\mathrm{c}(2 \times 8)$ reconstruction, electron energy ("start voltage" or SV) $=19.9 \mathrm{eV}$. (c) Schematic of the $\mathrm{c}(2 \times 8)$ reconstruction LEED pattern. (d) Ge surface after $\mathrm{C}$ deposition at $890^{\circ} \mathrm{C}, \mathrm{FOV}=$ $25 \mu \mathrm{m}$. (e) LEED pattern taken at $T=500{ }^{\circ} \mathrm{C}$ after $\mathrm{C}$ deposition showing first-order $\mathrm{Ge}(111)$ spots and a graphene diffraction ring, $\mathrm{SV}=35.5 \mathrm{eV}$. (f) LEED pattern with smaller aperture showing distinct graphene diffraction spots, $S V=35.5 \mathrm{eV}$. (g) Intensity of the graphene LEED pattern extracted from (e) shows random and uniform variations with orientation.

$\left(\sim 4 \times 10^{5} / \mathrm{cm}^{2}\right)$. The size and density of these defects increased with increasing sputtering cycles.

\section{Graphene on $\mathrm{Ge}(111)$}

In general, LEED indicated a high-quality Ge(111) surface after sputtering as evidenced by the well-known $\mathrm{c}(2 \times 8)$ reconstruction [Fig. 1(b)]. The $\mathrm{c}(2 \times 8)$ reconstruction is an ordered adatom phase with three equivalent domain orientations that 
result in the four 1/8-order satellite diffraction spots as labeled in Fig. 1(c). The $\mathrm{c}(2 \times 8)$ reconstruction exhibits a first-order transition at $300{ }^{\circ} \mathrm{C}$ to a disordered $(1 \times 1)$ phase. ${ }^{[10]}$

After surface preparation, the sample was heated and carbon was deposited. The LEEM image in Fig. 1(d) shows the surface after depositing carbon for $80 \mathrm{~min}$ at $890^{\circ} \mathrm{C}$. The surface contrast is clearly different from the pristine surface in Fig. 1(a). LEED obtained at high temperature [Fig. 1(e)] indicates the presence of a new diffraction ring with the spacing expected for graphene. Auger electron spectroscopy confirmed the presence of $\mathrm{C}$ on the surface after deposition (not shown). As shown in Fig. 1(g), the changes in LEED intensity along the ring are uniform with random variations. This suggests that the graphene islands nucleate in random orientations with equal probability on $\mathrm{Ge}(111)$. The illumination diameter for this area is $5 \mu \mathrm{m}$.

Reducing the illuminated area to $1.5 \mu \mathrm{m}$ in diameter results in the discrete graphene LEED spots as shown in Fig. 1(f). These distinct spots indicate that graphene domains with high crystalline quality exist. By counting the LEED spots and comparing their relative intensity, we estimate roughly that the aperture illuminated 10-20 graphene domains (assuming that the domains are of comparable size). Assuming a complete graphene layer (discussed below) gives an average grain size of $100-500 \mathrm{~nm}$, which is consistent with previous reports of growth by CVD. ${ }^{[7]}$
We were unable to effectively image the growth of individual graphene domains in real-time. LEEM requires a high voltage ( $15 \mathrm{kV}$ here) between the sample and the objective lens. Because growth is conducted at temperatures near the Ge melting point, any arcing caused by this high voltage often resulted in the destruction of the sample. Furthermore, imaging growth is complicated by the small graphene domain size and the low contrast between pristine Ge and graphene-covered Ge.

With a graphene layer covering the surface, it is not unreasonable to expect that it would significantly affect the $\mathrm{c}(2 \times 8)$ reconstruction. Surprisingly, this is not the case. Upon cooling, the $\mathrm{c}(2 \times 8)$ reconstruction forms as seen in the LEED pattern in Fig. 2(a). Indeed, as one sees in Fig. 2(b), the transition temperature of the graphene-covered surface is nominally only $10 \mathrm{~K}$ higher than pristine Ge(111). This is within the error of the temperature measurement. In principle there are two possible explanations for the unchanged transition temperature. The first is that the transition we observe occurs only in regions not covered by graphene. The second is that the relative free energies of the ordered and disordered phases are not affected significantly by the presence of graphene. We establish that the latter is true by showing that the disordered phase is different from that of the pristine surface over the entire sample.

The average intensities of the four 1/8-order $\mathrm{c}(2 \times 8)$ spots were measured while cooling at a rate of $-0.5 \mathrm{~K} / \mathrm{sec}$ for the

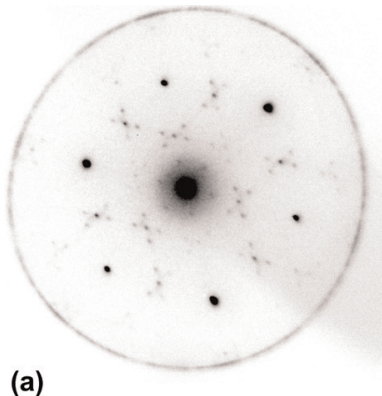

(a)

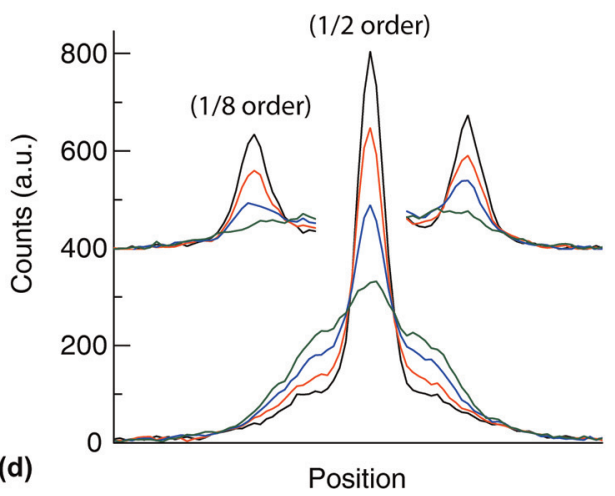

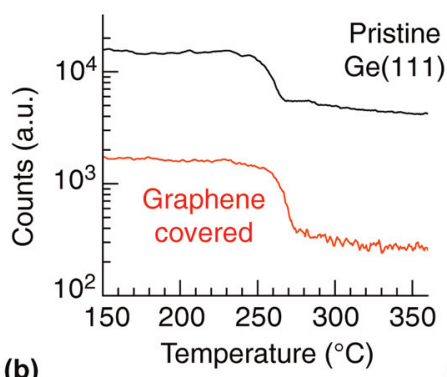

(c)

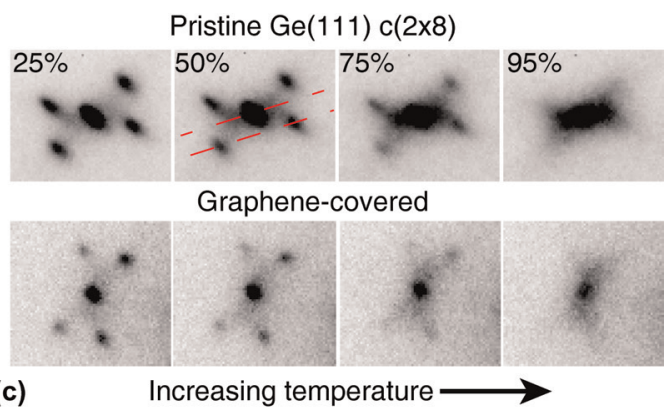

$c(2 \times 8) 1 / 8$ th order intensity change

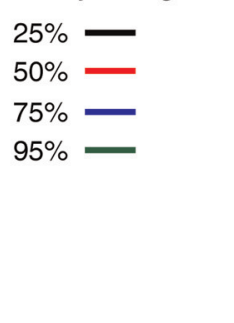

(e)

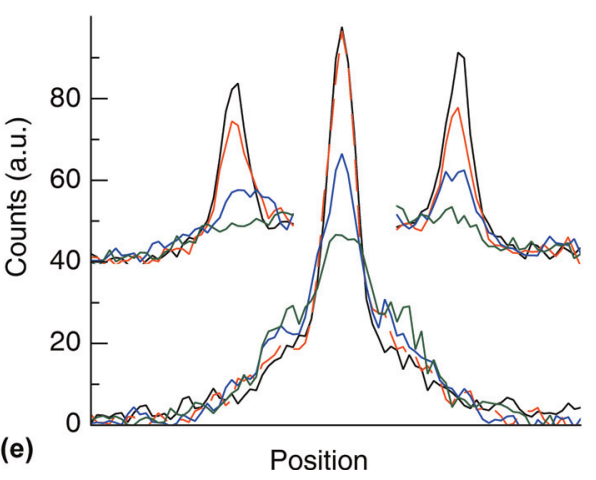

Figure 2. (a) Low temperature LEED pattern showing the presence of both graphene and $\mathrm{Ge}(111) \mathrm{c}(2 \times 8)$ reconstruction, $\mathrm{SV}=36.7 \mathrm{eV}$. (b) Comparison of the $c(2 \times 8)$ phase transition with temperature for pristine and graphene-covered $\mathrm{Ge}(111)$ by monitoring the $1 / 8$ th order $\mathrm{c}(2 \times 8)$ reconstruction beams. (c) Comparison of the evolution of $\mathrm{c}(2 \times 8)$ LEED spots with temperature for both pristine and graphene-covered $\mathrm{Ge}(111)$ compared at similar points through their respective changes in $c(2 \times 8)$ intensity, as determined by the intensity changes in (b) and denoted by the percentage value. (d), (e) Intensity profiles of $1 / 2$ and $1 / 8$-order $\mathrm{c}(2 \times 8)$ LEED spots taken along the red, dashed lines in $(\mathrm{c})$ and plotted together for pristine $\mathrm{Ge}(111) \mathrm{c}(2 \times 8)$ reconstruction (d) and the graphene-covered Ge surface (e) at various percentages of intensity change. 1/8-order intensity shifted in $y$-axis for clarity, scale is maintained. 
pristine and graphene-covered samples and are compared in Fig. 2(c) as a function of the LEED spot intensity change. Because of the transition temperature shift noted above, the change in intensity is used as a common metric, where the denoted intensity change is the percentage of the difference between the initial and final intensity of the $\mathrm{c}(2 \times 8)$ LEED spots [Fig. 2(b)]. From the LEED images in Fig. 2(c), one sees that, consistent with Ref. 10, the 1/2-order spot broadens significantly above the transition temperature for the pristine surface. This is confirmed by plotting its intensity taken along the red, dashed lines as the transition occurs [Fig. 2(d)]. The maximum intensity of the $1 / 2$-order spot decreases as it broadens. In contrast, the graphene-covered sample in Fig. 2(e) shows no broadening. Here, the maximum intensity begins decreasing at a point further along the transition. For the pristine surface, the intensity of the $1 / 2$ - and $1 / 8$-order spots monotonically decreases as the phase transition proceeds. Compared with the graphene-covered surface in Fig. 2(e), the 1/2-order spot maintains its initial intensity, while the $1 / 8$-order spots decrease in intensity by $50 \%$. In Ref. 10 , the broadening of the $1 / 2$-order spots is interpreted in terms of short-range order in the positions of $\mathrm{c}(2 \times 8)$ domain walls between ordered regions. Evidently graphene decreases this ordering. The measured differences in the diffraction pattern are independent of the LEED sampling location. We did not detect the broadening associated with the pristine surface anywhere on the graphenecovered sample. Thus, we conclude that the entire surface was covered by at least one monolayer of graphene. That the transition temperature is unchanged suggests that the $\mathrm{c}(2 \times 8)$ domain ordering at high temperature has negligible effect on the free energy of the disordered phase.

\section{Graphene on $\mathrm{Ge}(110)$}

Similar surface preparation and imaging conditions were used for $\mathrm{Ge}(110)$. The post-sputtered surface is shown in Fig. 3(a) along with its corresponding LEED pattern in Fig. 3(b). Compared with $\mathrm{Ge}(111)$, the $\mathrm{Ge}(110)$ surface is more complex. Multiple features have been reported, including uniformly spaced terraces forming $\left\{\begin{array}{lll}17 & 15 & 1\end{array}\right\}$ facets, a $\mathrm{c}(8 \times 10)$ reconstruction, and a $(16 \times 2)$ reconstruction. ${ }^{[1,21,22]}$. These features are stable in specific temperature windows and their formation can be kinetically limited. ${ }^{[21]}$ The LEED pattern in Fig. 3(b) has spots with half the periodicity of the first-order spots (red circles) in the [001] direction and one-eighth the periodicity in the [111] direction [see Fig. 3(d) for reference], which have been previously attributed to $\left\{\begin{array}{lll}17 & 15 & 1\end{array}\right\}$ facets. ${ }^{[1]} \mathrm{We}$ note that these facets may occur in the [779] direction as well; however, facets oriented along a single direction, as seen here, have been previously reported for miscuts less than $2^{\circ} \cdot .^{11]}$

Figure 3(c) shows the surface after depositing $\mathrm{C}$ for $45 \mathrm{~min}$ at $870{ }^{\circ} \mathrm{C}$. The surface exhibits similar features as those seen on graphene-covered $\mathrm{Ge}(111)$, and the presence of graphene is confirmed by the LEED pattern in Fig. 3(d). The graphene exhibits significantly improved alignment compared with
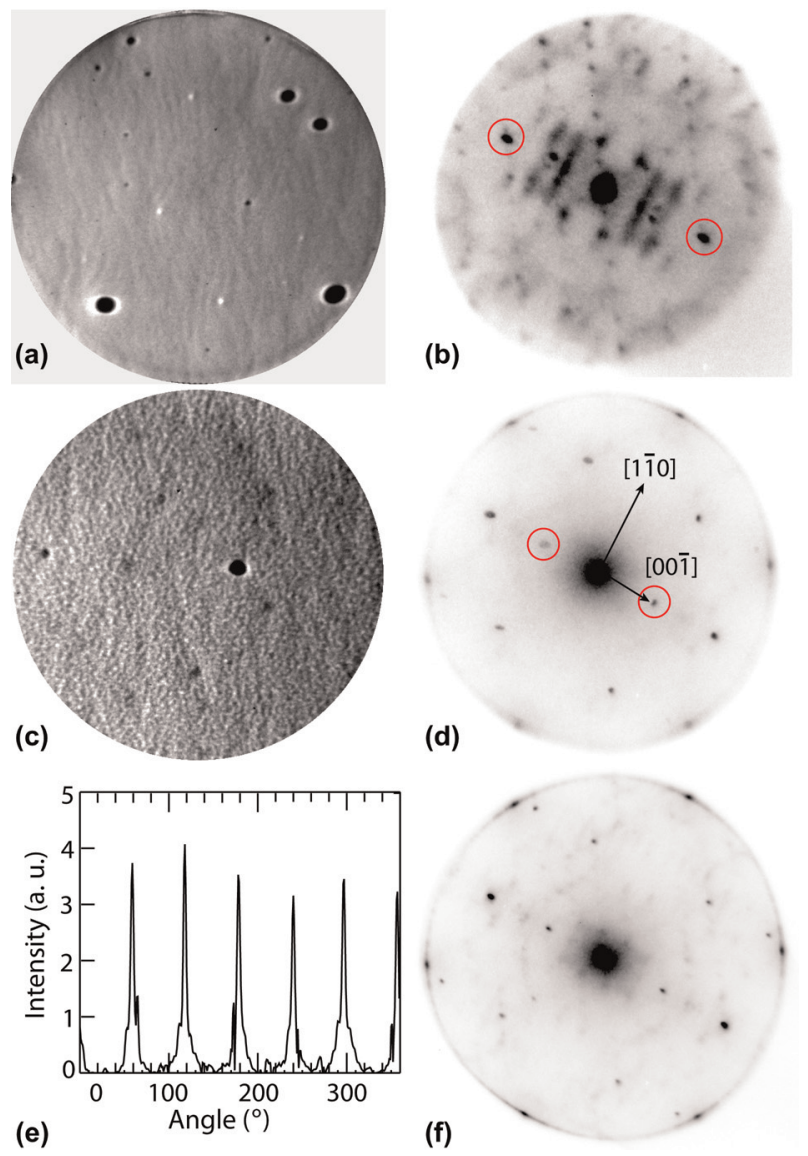

(d)
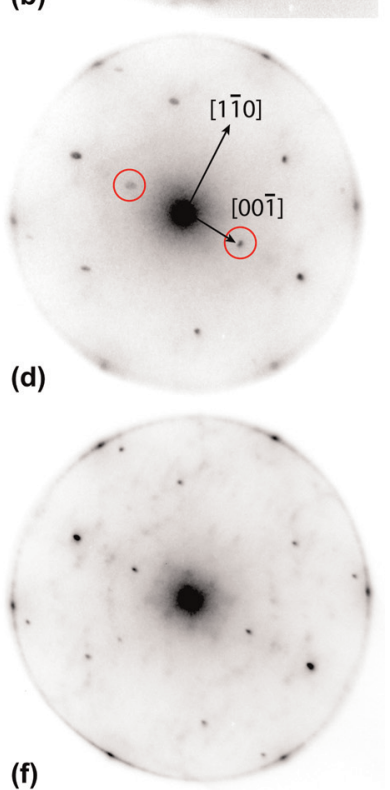

Figure 3. (a) LEEM image of pristine $\mathrm{Ge}(110)$ surface; FOV $=15 \mu \mathrm{m}$. (b) LEED pattern of pristine surface. First-order spots highlighted by red circles, superstructure spots attributed to $\{17151\}$ facets; $S V=11.9 \mathrm{eV}$. (c) LEEM image after $\mathrm{C}$ deposition for $45 \mathrm{~min}$ at $870^{\circ} \mathrm{C}$; FOV $=15 \mu \mathrm{m}$. (d) LEED pattern after $\mathrm{C}$ deposition showing localized graphene diffraction spots. For reference, red circles highlight the same spots in (b). $T=870^{\circ} \mathrm{C}, \mathrm{SV}=38.1$ $\mathrm{eV}$. (e) Intensity of the graphene LEED pattern extracted from (d) shows that the graphene is strongly localized to a single orientation. (f) LEED pattern after cooling to $90^{\circ} \mathrm{C}, \mathrm{SV}=37.3 \mathrm{eV}$.

graphene on Ge(111). As shown in Fig. 3(e), diffraction from the graphene does not result in a ring but is localized to within $10^{\circ}$ of a preferred orientation. This preferred orientation is along the $\mathrm{Ge}[1 \overline{1} 0]$ direction of the $\mathrm{Ge}(110)$ surface, that is, the graphene lattice vectors align with Ge[110]. Here, an illumination aperture with a $10 \mu \mathrm{m}$ diameter was used and the LEED pattern did not change with decreasing aperture size. The same preferred alignment was seen as the illumination region was scanned across the surface. Previous work has suggested that the hydrogen termination of Ge induces graphene alignment on $\mathrm{Ge}(110) \cdot{ }^{[7]}$ Because our growth by PVD occurs in the absence of hydrogen, our results show that hydrogen is not responsible for the preferred graphene domain alignment on the (110) surface.

In contrast to $\mathrm{Ge}(111)$, the graphene-covered surface is characterized by a significantly different LEED pattern [Fig 3(f)] 
than that of the pristine surface upon cooling. The $\left\{\begin{array}{lll}17 & 15 & 1\end{array}\right\}$ facets are nearly extinguished - the faint appearance of features misoriented relative to the facets seen in Fig. 3(b) are attributed to the facets in the [779] direction. Clearly the Ge(110) surface is affected by the presence of graphene. The extinguished faceting seen in LEED was observed uniformly across the sample. Thus, we conclude that, at a minimum, a full graphene monolayer is present.

\section{Water liftoff and Raman results}

While growth on Ge wafers provides significant advantages for process integration, the graphene film must still be separated from the Ge substrate because Ge is a good electrical conductor at room temperature due to its relatively small band gap-less than $0.7 \mathrm{eV}$. Previous efforts achieved liftoff from Ge by depositing a thin layer of $\mathrm{Au}$ on the graphene and then peeling off the gold-graphene film. ${ }^{[7]}$ Then, the gold film was removed by chemical etching, a process that can result in significant deterioration of graphene's electrical performance. ${ }^{[23]}$

We propose a less invasive transfer technique that utilizes the water-soluble nature of germanium oxide. ${ }^{[24]}$ Figure 4(a) shows an optical microscopy image of fragments collected

(a)
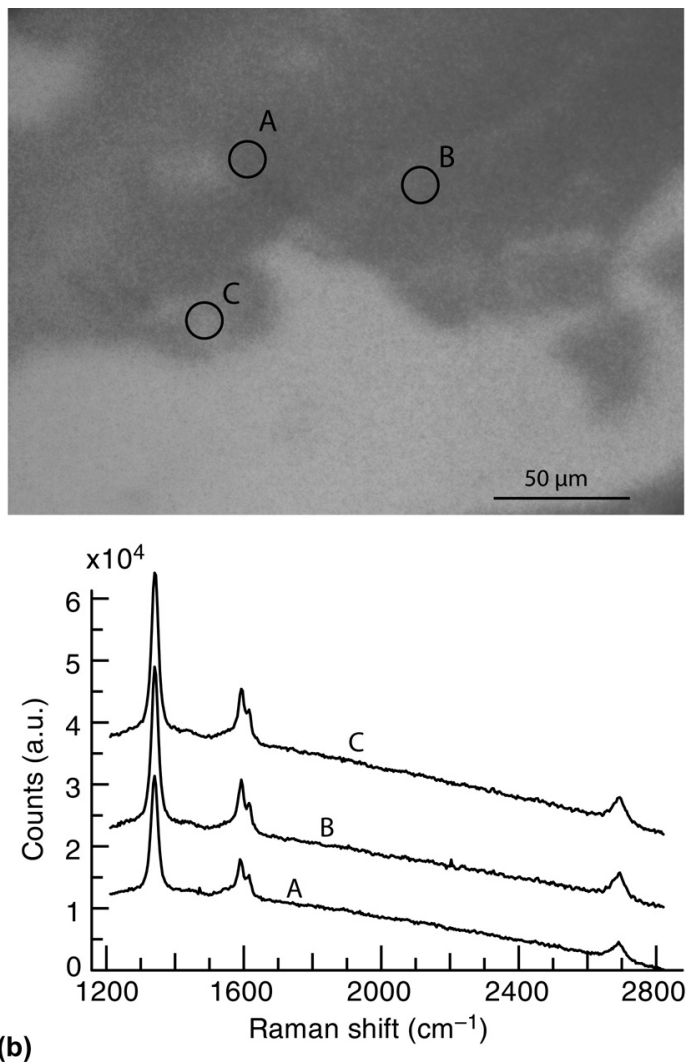

Figure 4. (a) Optical microscopy image of a graphene flake on $\mathrm{SiO}_{2}$ wafer after soaking a graphene-covered $\mathrm{Ge}(110)$ sample in water and collecting the flakes with the wafer. (b) Raman spectra taken at the three labeled locations in (a). The Raman peaks are consistent with defective graphene. after soaking a sample of graphene grown on $\mathrm{Ge}(110)$ in water. The micro-Raman spectra shown in Fig. 4(b) were taken from the three spots labeled in Fig. 4(a). The Raman spectra exhibit strong $\mathrm{D}$ and $\mathrm{G}$ peaks, and small 2D peaks, which is consistent with defective graphene.

It is likely that the underlying germanium surface oxidized after removal from the ultra-high vacuum LEEM environment, especially given the defective nature of the film (which may facilitate oxygen penetration). Because germanium oxide is water soluble, we speculate that the surface oxide dissolves during water soaking, resulting in the release of the graphene film. This technique is supported by previous efforts (not shown here), where multilayer graphene films of millimeter dimensions were transferred using only water. We expect monolayer films to be transferable by optimizing this process.

The graphene we have grown on $\mathrm{Ge}(110)$ has a small spread of angles $\left[10^{\circ}\right.$, Fig. 3(e)], causing grain boundaries in the completed graphene film. Due to the small grain size, the grain boundary density is likely high and can account for the low quality detected by Raman spectroscopy.

\section{DFT calculations of the structure of graphene on $\mathrm{Ge}(110)$ and $\mathrm{Ge}(111)$}

To investigate the differences in the alignment, we have calculated the structure of graphene on the two substrates by DFT. Our focus is on the role of the adatom structure in graphene binding and morphology. We start by considering the ordered low-temperature structures as they have been well characterized. For the Ge(110) surface, we studied the $c(8 \times 10)$ reconstruction for computational convenience: the unit cell size best matches graphene. Since the adatom structure of the $c(16 \times 2)$ reconstruction is similar, we expect similar graphene-adatom interactions.

Reconstructed surfaces of $\mathrm{Ge}(111) \mathrm{c}(2 \times 8)$ and $\mathrm{Ge}(110) \mathrm{c}$ $(8 \times 10)$ were constructed by adding adatoms at positions given by previous experimental and theoretical studies ${ }^{[25,26]}$ to bulk truncated surfaces created from the optimized cubic close-packed structure $(a=b=c=5.763 \AA)$. The reconstructed surfaces were then relaxed until all forces were less than 0.02 $\mathrm{eV} / \AA$. The graphene sheet was then stretched or compressed over the Ge substrate in an effort to minimize lattice strain. The graphene sheet was stretched $1.87 \%$ and compressed $0.65 \%$ on the $\mathrm{Ge}(111) \mathrm{c}(2 \times 8)$ surface in the $a$ and $b$ directions, respectively. On the $\mathrm{Ge}(110) \mathrm{c}(8 \times 10)$ surface, a stretch of $3.4 \%$ in both directions was required. [Calculations were carried out with graphene compressed by $4 \%$ but the graphene sheet buckled. The corrugation amplitude would likely be larger if there was no lattice mismatch because stretching the graphene sheet makes it more rigid. Since the corrugation amplitude predicted on $\mathrm{Ge}(110)$ is nearly an order of magnitude larger than that on $\mathrm{Ge}(111)$, the reported results are believed to be at least qualitatively correct.] The resulting optimized structures and pertinent structural information are shown in Fig. 5. The binding and graphene deformation energies per carbon atom 

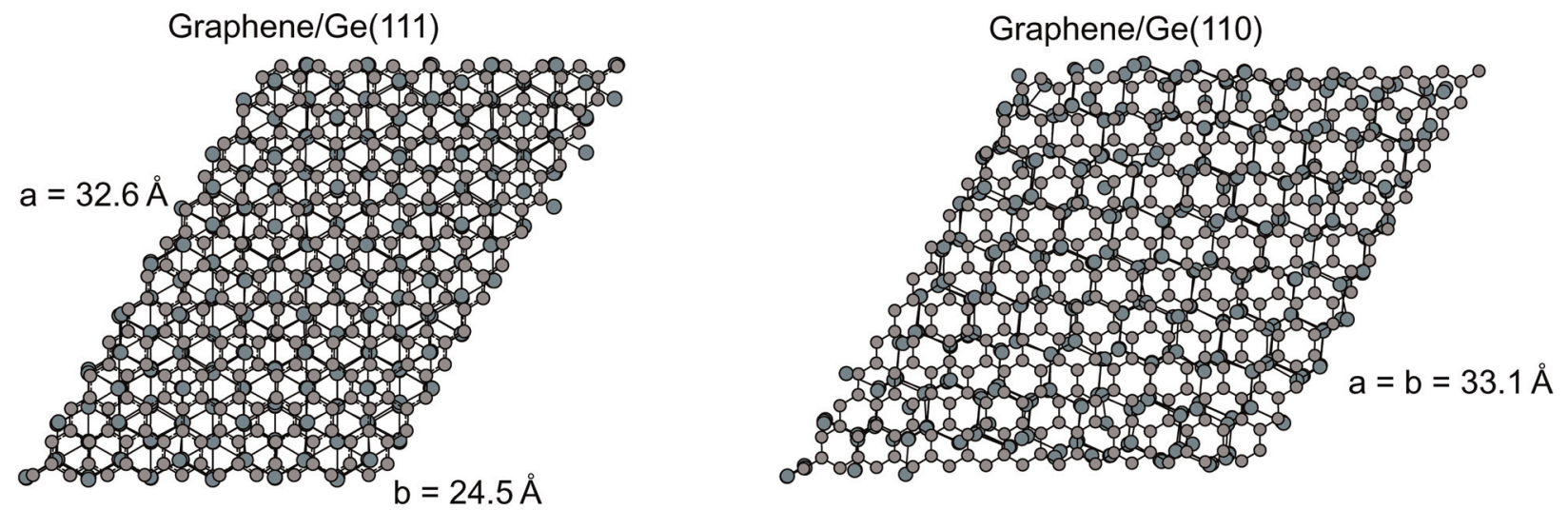

(a)

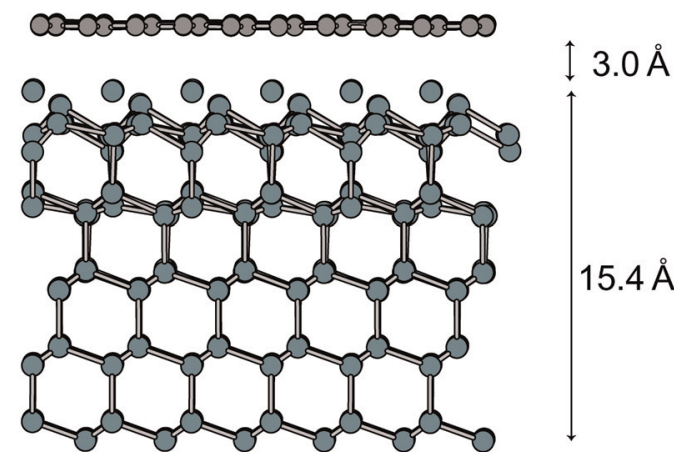
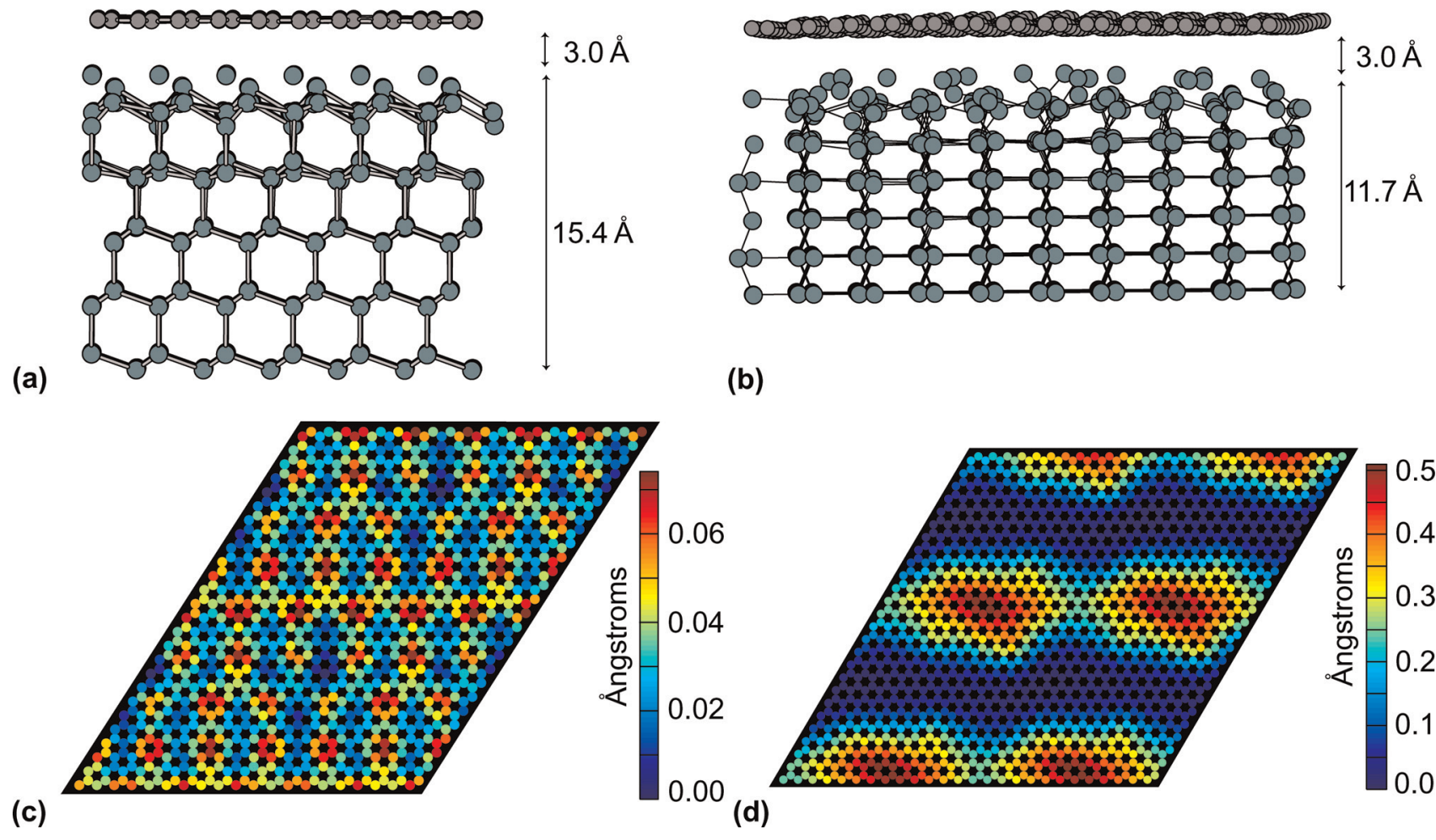

(b)

Figure 5. Top and side views of DFT optimized graphene on $(\mathrm{a}) \mathrm{Ge}(111) \mathrm{c}(2 \times 8)$ and $(\mathrm{b}) \mathrm{Ge}(110) \mathrm{c}(8 \times 10)$ structures and relevant geometric parameters. Carbon atoms are grey and Ge atoms are green. (c), (d) Top view of graphene corrugations on the $\mathrm{Ge}(111) \mathrm{c}(2 \times 8)$ surface $(\mathrm{c})$ and the $\mathrm{Ge}(110) \mathrm{c}(8 \times 10)$ surface $(\mathrm{d})$. The colored scale bar indicates the distance in the z-direction between the $\mathrm{C}$ atom and the plane of the Ge surface adatoms, i.e., the height difference.

of the two systems were determined as follows:

$$
\begin{gathered}
E_{\mathrm{BE}}=\frac{E_{\mathrm{G} / \mathrm{Ge}}-E_{\mathrm{G}}-E_{\mathrm{Ge}}}{N} \\
E_{\mathrm{def}}=\frac{E_{\mathrm{G}-\text { def }}-E_{\mathrm{G}}}{N}
\end{gathered}
$$

where $E_{\mathrm{G} / \mathrm{Ge}}$ is the total energy of the system, $E_{\mathrm{G}}$ is the total energy of the optimized isolated graphene layer (in the respective Ge surface unit cell), $E_{\mathrm{G}-\text { def }}$ is the total energy of the graphene sheet in the G/Ge geometry, $E_{\mathrm{Ge}}$ is the total energy of the Ge surface, and $N$ is the number of carbon atoms. The resulting graphene binding energies on the $\mathrm{Ge}(111) \mathrm{c}(2 \times 8)$ and $\mathrm{Ge}$ $(110) \mathrm{c}(8 \times 10)$ surfaces are -40 and $-37 \mathrm{meV}$ per $\mathrm{C}$ atom, respectively. The relatively weak interaction can be attributed to the Ge adatoms; although the graphene sheet is relatively close to the adatoms $(\sim 3 \AA)$, on average the sheet lies 3.7-3.8 above the Ge surface atoms in both cases, slightly above the typical van der Waals distance. The predicted graphene deformation energies on the $\mathrm{Ge}(111) \mathrm{c}(2 \times 8)$ and $\mathrm{Ge}(110) \mathrm{c}(8 \times 10)$ 
surfaces are 0.61 and $1.63 \mathrm{meV} / \mathrm{C}$, respectively. The difference in energy correlates directly to the difference in the observed corrugation patterns/amplitudes since graphene on $\mathrm{Ge}(110)$ has corrugations that are an order of magnitude greater than on Ge(111) [see Fig. 5(c) and 5(d)].

We attribute the extremely small corrugations of $\mathrm{Ge}(111)$ to the fact that graphene is stiff and cannot respond to the relatively short wavelength corrugations corresponding to the adatomadatom separations $(\sim 8 \AA)$ of the $\mathrm{c}(2 \times 8)$ reconstruction. At the temperatures of the growth experiments, the reconstructions modeled here are disordered. However, graphene on Ge(111) is likely to remain flat because the average adatom separation is not expected to change significantly. For example, the adatom density on $\mathrm{Si}(111)$ is approximately temperature independent despite having a high-temperature " $1 \times 1$ " phase and multiple metastable reconstructions. ${ }^{[27]}$ To provide further support, we repeated the DFT simulations using a perturbed $\mathrm{c}(2 \times 8)$ ground state to mimic the disordered state. Rows of adatoms were shifted with respect to each other by one Ge lattice constant along the $b$ direction, labeled in Fig. 5(a). These shifts avoid the occupancy of nearest neighbor adatom binding sites and generate three additional variations of the $\mathrm{c}(2 \times 8)$ unit cell. We find that the graphene structure remains essentially identical-with changes in corrugation and average height less than $0.1 \AA$.

The independence of graphene structure on adatom configuration suggests that its binding energy will also not depend sensitively on the adatom configuration, thus explaining why the presence of graphene does not significantly affect the disordering temperature of the $\mathrm{c}(2 \times 8)$ reconstruction. Indeed, the calculated binding energy of graphene on the perturbed structures is the same to within $4 \mathrm{meV}$ per Ge atom, which is much less than $k T_{c}$ of the $\mathrm{c}(2 \times 8)$ ordering transition $(50 \mathrm{meV})$. [However, these perturbations raise the energy of the bare Ge surface significantly (by as much as $200 \mathrm{meV}$ per $\mathrm{c}(2 \times 8)$ unit cell), consistent with the observed $\mathrm{c}(2 \times 8)$ ground state.]

\section{Discussion}

The domain size and the significantly improved rotational order of graphene on $\mathrm{Ge}(110)$ compared with $\mathrm{Ge}(111)$ is consistent with previous reports of graphene grown by CVD. ${ }^{[7]}$ However, our results call into question some of the conclusions obtained in Ref. 7. The authors suggest that hydrogen plays a critical role in obtaining the orientation preference. Here, growth occurred in complete absence of hydrogen gas and highly oriented films are still obtained. Instead, our DFT calculations suggest an alternative reason for the poor alignment on $\mathrm{Ge}(111)$. For the energy of an incommensurate graphene film to depend on orientation, it must be corrugated. ${ }^{[6]}$ So the lack of corrugation in the DFT calculations for Ge(111) suggests that there is no energetically preferred alignment. This is consistent with our finding that the graphene binding energy does not depend on the adatom configuration of $\mathrm{Ge}(111)$. The reasons for good alignment on $\mathrm{Ge}(110)$ is less clear. While the $\mathrm{c}(8 \times 10)$ reconstruction gives a large corrugation of the graphene sheet, it is unclear if this corrugation would persist to high temperature. It is possible that the relatively small number of adatoms on $\mathrm{Ge}(110)$ are still grouped pentagons at high temperature, which would result in large average separations between pentagons and allow the graphene lattice to become corrugated with an energy which is sensitive to orientation. But regardless of the corrugation, that the low-temperature surface reconstruction of $\mathrm{Ge}(110)$ changes due to graphene's presence suggests a stronger dependence of the interaction with the structure (and thus orientation) of the substrate.

\section{Conclusion}

We find that graphene grown on $\mathrm{Ge}(110)$ by PVD has fewer rotational domains than growth on Ge(111), consistent with previous CVD results. The surface underneath graphene for both terminations is reconstructed. The insignificant change in disordering temperature of the $\mathrm{Ge}(111) \mathrm{c}(2 \times 8)$ adatom reconstruction underneath graphene compared with clean $\mathrm{Ge}$ indicates a very weak interaction. This is supported by DFT calculations, which show that graphene is not significantly corrugated by the reconstruction. On the other hand, the reconstruction underneath graphene on $\mathrm{Ge}(110)$ is strongly affected by the graphene, indicating a more significant interaction with the adatoms. Indeed, DFT shows that graphene on reconstructed $\mathrm{Ge}(110)$ is strongly corrugated. We suggest that the absence of corrugation on reconstructed $\mathrm{Ge}(111)$ is responsible for the poor rotational alignment compared with $\mathrm{Ge}(110)$.

\section{Acknowledgment}

Work performed at Sandia National Laboratories was supported by the Director, Office of Science, Office of Basic Energy Sciences, Division of Materials Sciences and Engineering. Sandia National Laboratories is a multiprogram laboratory operated by Sandia Corporation, a Lockheed-Martin Company, for the U.S. Department of Energy under Contract No. DE-AC04-94AL85000. ODD, PCR, and JMW were supported by the NSF under Grant No. DMR-1105541. Development of the graphene transfer process was funded by the Director, Office of Science, Office of Basic Energy Sciences, Material Sciences and Engineering Division of the U.S. Department of Energy under Contract No. DE-AC02-05CH11231.

\section{References}

1. Q. Yu, L.A. Jauregui, W. Wu, R. Colby, J. Tian, Z. Su, H. Cao, Z. Liu, D. Pandey, D. Wei, T.F. Chung, P. Peng, N.P. Guisinger, E.A. Stach, J. Bao, S.S. Pei, and Y.P. Chen: Control and characterization of individual grains and grain boundaries in graphene grown by chemical vapor deposition. Nat. Mater. 10, 443 (2011).

2. S. Nie, N.C. Bartelt, J.M. Wofford, O.D. Dubon, K.F. McCarty, and K. Thürmer: Scanning tunneling microscopy study of graphene on $A u$ (111): growth mechanisms and substrate interactions. Phys. Rev. B 85, 205406 (2012).

3. S. Nie, J.M. Wofford, N.C. Bartelt, O.D. Dubon, and K.F. McCarty: Origin of the mosaicity in graphene grown on Cu(111). Phys. Rev. B 84, 155425 (2011). 
4. E. Loginova, S. Nie, K. Thürmer, N.C. Bartelt, and K.F. McCarty: Defects of graphene on $\operatorname{Ir}(111)$ : rotational variants and ridges. Phys. Rev. B 80, 085430 (2009).

5. H. Tetlow, J. Posthuma de Boer, I.J. Ford, D.D. Vvedensky, J. Coraux, and L. Kantorovich: Growth of epitaxial graphene: theory and experiment. Phys. Rep. 542, 195 (2014).

6. P.C. Rogge, K. Thürmer, M.E. Foster, K.F. McCarty, O.D. Dubon, and N.C. Bartelt: Real-time observation of epitaxial graphene domain reorientation. Nat. Commun. 6, 6880 (2015).

7. J.H. Lee, E.K. Lee, W.J. Joo, Y. Jang, B.S. Kim, J.Y. Lim, S.H. Choi, S.J. Ahn, J.R. Ahn, M.H. Park, C.W. Yang, B.L. Choi, S.W. Hwang, and D. Wang: Wafer-scale growth of single-crystal monolayer graphene on reusable hydrogen-terminated germanium. Science 344, 286 (2014).

8. G. Wang, M. Zhang, Y. Zhu, G. Ding, D. Jiang, Q. Guo, S. Liu, X. Xie, P.K. Chu, Z. Di, and X. Wang: Direct growth of graphene film on germanium substrate. Sci. Reports 3, 2465 (2013).

9. G. Lippert, J. Dabrowski, T. Schroeder, M.A. Schubert, Y. Yamamoto, F. Herziger, J. Maultzsch, J. Baringhaus, C. Tegenkamp, M.C. Asensio, J. Avila, and G. Lupina: Graphene grown on $\mathrm{Ge}(001)$ from atomic source. Carbon 75, 104 (2014).

10. R.J. Phaneuf and M.B. Webb: A LEED study of Ge(111): a hightemperature incommensurate structure. Surf. Sci. 164, 167 (1985).

11. B.Z. Olshanetsky, S.M. Repinsky, and A.A. Shklyaev: LEED investigation of germanium surfaces cleaned by sublimation of sulphide films; structural transitions of clean Ge(110) surface. Surf. Sci. 64, 224 (1977).

12. J. Klimeš, D.R. Bowler, and A. Michaelides: Van der Waals density functionals applied to solids. Phys. Rev. B 83, 195131 (2011).

13. G. Kresse and J. Furthmüller: Efficiency of ab-initio total energy calculations for metals and semiconductors using a plane-wave basis set. Comp. Mater. Sci. 6, 15 (1996).

14. G. Kresse and J. Furthmüller: Efficient iterative schemes for ab initio totalenergy calculations using a plane-wave basis set. Phys. Rev. B 54, 11169 (1996).

15. G. Kresse and J. Hafner: Ab initio molecular dynamics for liquid metals. Phys. Rev. B 47, 558 (1993).

16. G. Kresse and J. Hafner: Ab initio molecular-dynamics simulation of the liquid-metal-amorphous-semiconductor transition in germanium. Phys. Rev. B 49, 14251 (1994).

17. P. Janthon, F. Viñes, S.M. Kozlov, J. Limtrakul, and F. Illas: Theoretical assessment of graphene-metal contacts. J. Chem. Phys. 138, 244701 (2013).

18.Z. Wang, S.M. Selbach, and T. Grande: Van der Waals density functional study of the energetics of alkali metal intercalation in graphite. RSC Adv. 4, 4069 (2014).

19. E. Bauer: Low energy electron microscopy. Rep. Prog. Phys. 57, 895 (1994).

20.M.S. Altman: Trends in low energy electron microscopy. J. Phys.: Condens. Matter. 22, 084017 (2010).

21. T. Ichikawa: In-situ STM observations of ordering behaviors on $\mathrm{Ge}(110)$ surfaces and atomic geometry of the Ge\{17 151$\}$ facet. Surf. Sci. $\mathbf{5 6 0}$, 213 (2004).

22. C.H. Mullet and S. Chiang: Reconstructions and phase transition of clean Ge(110). Surf. Sci. 621, 184 (2014).

23. A. Pirkle, J. Chan, A. Venugopal, D. Hinojos, C.W. Magnuson, S. McDonnell, L. Colombo, E.M. Vogel, R.S. Ruoff, and R.M. Wallace: The effect of chemical residues on the physical and electrical properties of chemical vapor deposited graphene transferred to $\mathrm{SiO}_{2}$. Appl. Phys. Lett. 99, 122108 (2011).

24. M.K. Murphy and H. Hill: Studies in germanium oxide systems: III, solubility of Germania in water. J. Am. Ceram. Soc. 48, 109 (1965).

25. T. Ichikawa: Atomic geometry of the $\mathrm{Ge}(110) \mathrm{c}(8 \times 10)$ structure. Surf. Sci. 560, 205 (2004)

26. L. Patthey, E.L. Bullock, and K. Hricovini: The atomic geometry of the Ge (111) surface as a function of temperature studied by photoemission and photoelectron diffraction. Surf. Sci. 269-270, 28 (1992).

27. Y.N. Yang and E.D. Williams: High atom density in the " $1 \times 1$ " phase and origin of the metastable reconstructions on Si(111). Phys. Rev. Lett. 72, 1862 (1994). 